\title{
Defect depth estimation using magneto optical imaging with magnetophotonic crystal
}

\author{
R. Hashimoto, T. Yonezawa, H. Takagi, T. Goto, H. Endo*, A. Nishimizu* and M. Inoue \\ Department of Electrical and Electronic Information Engineering, Toyohashi Univ. of Tech., 1-1 Hibarigaoka, Tempaku-cho, \\ Toyohashi, Aichi 441-8580, Japan \\ *Hitachi Research Laboratory, Hitachi Ltd., Hitachi, Ibaraki 319-1221, Japan
}

\begin{abstract}
Non-destructive inspection (NDI) is used to detect defects for safety. The magneto optical (MO) imaging is an attractive method for NDI. The MO imaging can visualize defects as a light intensity of image, which depends on the polarization rotation angle of the MO film. The polarization rotation angle depends on the stray filed in the vertical direction. To accurately detect the stray field, it is necessary to enhance the Faraday rotation angle. Therefore, we focused on magnetophotonic crystal (MPC), which can enhance the MO effect with thin film, comprising the polycrystalline bismuth substituted yttrium iron garnet. We estimated the defect depth with the MPC located on the iron with a defect, which depth was varied from $1 \mathrm{~mm}$ to $10 \mathrm{~mm}$. The results showed good agreement with the defect depth measured by the dial gauge.
\end{abstract}

Key words: non-destructive inspection, magnetophotonic crystal, Faraday effect, magneto optical imaging, thin film

\section{Introduction}

Non-destructive inspection is used to find the defects in the parts of the constructions for safety ${ }^{1)}$. It is difficult to estimate the defect depth from optical imaging because it can detect only 2-dimensional information. In particular, the magneto optical (MO) imaging ${ }^{2-4)}$ is useful for the high speed detection ${ }^{5)-6}$ of a metal surface cracks. The magnetization direction of the MO medium (MO film) is changed perpendicularly which effect on the Faraday rotation by the stray field generated at the edge of the crack. The Faraday rotation angle is converted into brightness of the image through the polarizer arranged in a Cross-Nicol alignment. This is the principle of the MO imaging.

The defect depth is estimated via contrast of the MO image $^{7)}$, because stray field strength depends on the defect depth. Therefore, to realize the high-accurate depth estimation, the high-contrast MO image is required. For the high-contrast MO image, we used magnetophotonic crystal (MPC)8), comprising the polycrystalline garnet film sandwiched between two Bragg mirrors. The MPC can enhance the Faraday rotation angle with the thin magnetic layer, i.e., can enhance the contrast of the MO image. Previously, we demonstrated the MO imaging using the MPC and obtained the surface image of defects from MO image ${ }^{9}$.

In this study, we estimated the defect depth using MO imaging with the MPC located on the specimen. The estimated results showed good agreement with the defect depth measured by the dial gauge.

\section{Fabrication of the magnetophotonic crystal}

The MO films are required to have a large Faraday rotation angle to obtain high-contrast MO image. The
MPC can provide a large rotation angle due to the Fabry-Perot resonance in the thin MO film. Therefore, the MPC is valuable for detection of defect by MO imaging. The MPC fabrication process of previously work ${ }^{10)}$ is shown below. We used the polycrystalline bismuth substituted yttrium iron garnet (Bi:YIG) film for the defect layer of the MPC, where the optical wave is localized. The Bi:YIG was deposited by the radio-frequency ion beam sputtering. The Bragg mirrors were composed of $\mathrm{SiO}_{2}$ and $\mathrm{Ta}_{2} \mathrm{O}_{5}$ films deposited by electron beam evaporation. The MPC was comprising of substituted gadolinium gallium garnet $(\mathrm{SGGG})$ substrate / $\left(\mathrm{Ta}_{2} \mathrm{O}_{5} / \mathrm{SiO}_{2}\right)^{2}$ pair / (Bi:YIG) $)^{350} \mathrm{~nm} /$ $\left(\mathrm{SiO}_{2} / \mathrm{Ta}_{2} \mathrm{O}_{5}\right)^{4}$ pair. Figure 1 shows the Faraday rotation angle of fabricated MPC and Bi:YIG single film deposited on the SGGG substrate. The Bi:YIG film thickness was $350 \mathrm{~nm}$ and this is same as the defect layer thickness of MPC. The Faraday rotation angle of MPC was more than 5 times larger $(8.5$ deg.) than that of the Bi:YIG single film. Therefore, the result indicated a possibility to the high-accuracy depth estimation by the MPC.

\section{Experimental method}

The MO images of the defects were obtained by placing a MPC on the surface of the carbon steel (specimen). The schematic of the MO imaging system is shown in Fig. 2. The spatial resolution of the MO imaging system depends on the magnetic domain size of the MO film. We used the polycrystalline magnetic garnet ${ }^{10)}$ as a MO film. The spatial resolution of the MO imaging system can be estimated about $50 \mathrm{~nm}$ via small domain sizes of the magnetic film.

The static magnetic field was applied to the specimen by the magnetic yoke. The saturation 
magnetization of the specimen was about $50 \mathrm{G}$ and the relative permeability $\left(\mu_{\mathrm{r}}\right)$ was 40 . The specimen had a 1 $\mathrm{mm}$ width cylindrical hole as a defect.

The applied magnetic field was 220 Oe which is approximately linear part limit of hysteresis. Because the MPC shows hysteresis loop in Fig. 1. Therefore, we used only linear part in the hysteresis of the MPC. The Faraday rotation angle was converted into the light intensity through a polarizing plate. The Faraday rotation angle depends on the stray filed in the vertical direction. The stray field became stronger depending on the defect depth. Therefore, the defect depth can be estimated by the light intensity of the MO image. The light intensity was obtained by the charge-coupled device (CCD) camera. Light source was the xenon lamp of $150 \mathrm{~W}$. The wavelength of the light source was 546 $\mathrm{nm}$ which corresponded to the localized mode of the MPC.

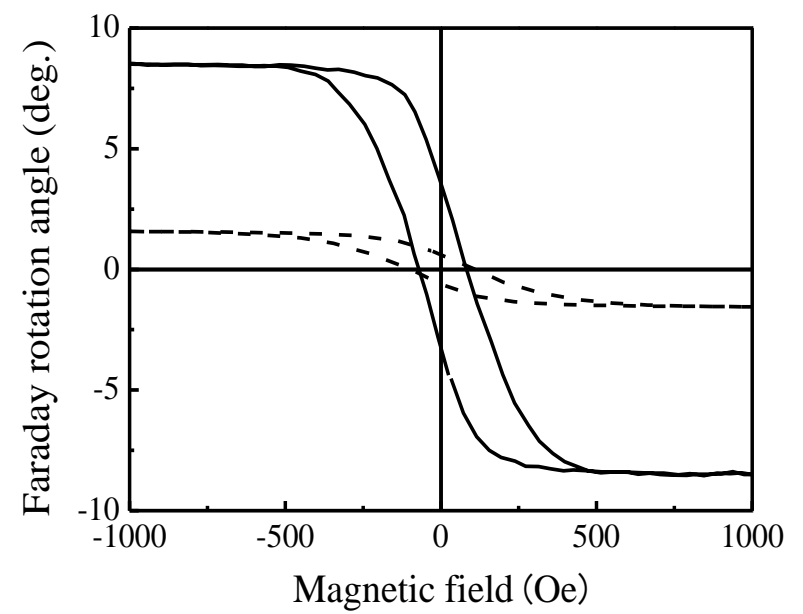

Fig. 1 Faraday rotation angle of MO films. Solid line is rotation angle of MPC. Dashed line is rotation angle of Bi:YIG single film. The film thickness is the same as the magnetic layer thickness of the MPC (350 nm).

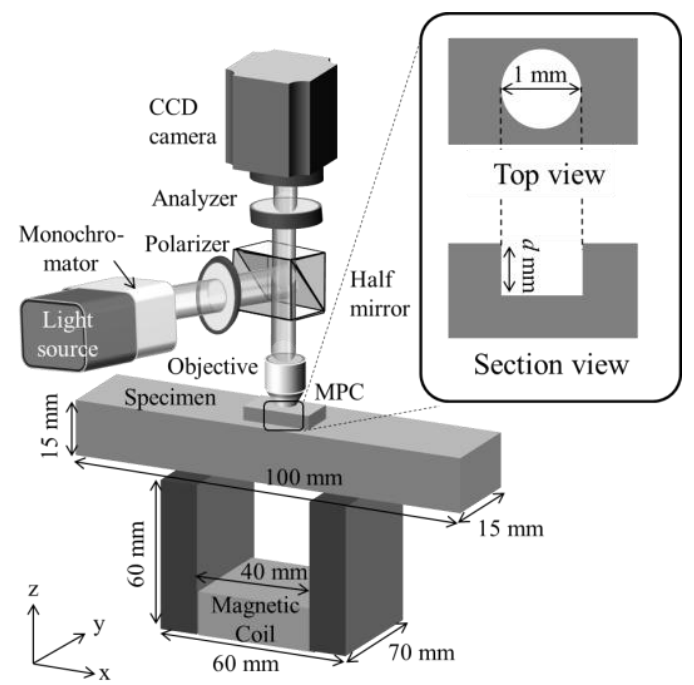

Fig. 2 The schematic of the MO imaging system.

\section{Results and discussion}

The obtained MO image from the $1 \mathrm{~mm}$ depth defect is shown in Fig. 3 (a). The MPC can enhance the Faraday rotation angle. Therefore, the contrast of the MO image is sufficient value. The relationship between light intensity and position on a white line in Fig. 3 (a) is shown in Fig. 3 (b) We estimated the defect depth from the maximum light intensity value (Peak value). Figure 4 shows the depth dependence of the peak values of the light intensity from the MO image. The error bars in Fig. 4 show the standard deviation of 10 measurement results. The peak value becomes larger by increase of the defect depth. However, the peak value was saturated over $5 \mathrm{~mm}$ depth. Therefore, in order to estimate the defect depth, it is necessary to obtain the depth dependence of the stray field.

To estimate the defect depth, we calculated the defect depth dependence of the stray field. The perpendicular stray field $\left(H_{\mathrm{z}}\right)$ from the defect was calculated with the finite element simulator (COMSOL Multiphysics 4.4). A square pole of iron $\left(\mu_{\mathrm{r}}=40\right)$ was defined as a specimen in the simulation model. The specimen had a cylindrical hole as a defect. The diameter of the defect was $1 \mathrm{~mm}$ and the depth $(d)$ of the defect was varied from $1 \mathrm{~mm}$ to $10 \mathrm{~mm}$. Figure 5 shows a schematic of the simulation model. We designed the simulation model enable to fabricate easily in order to compare with the experimental results. The simulation model was set on the magnetic yoke to apply magnetic field of 220 Oe. The specimen and the magnetic yoke were surrounded by air $\left(\mu_{\mathrm{r}}=1\right)$. An exterior boundary condition was zero magnetic scalar potential.

The stray field was calculated by the finite element simulator. To convert the stray field into the light intensity, we obtained the relationship between the light intensity and the magnetic field experimentally. The light intensity was measured from the MO imaging system with the Helmholtz coil. When magnetic field applied with Helmholtz coil was same as calculated stray field. In this way, the calculated stray field was converted to the experimental light intensity.

The solid line in Fig. 4 shows the simulation results. The simulation results showed good agreement with the experimental results. Then, we calculated the applied field intensity to estimate the defect depth from $1 \mathrm{~mm}$ to $10 \mathrm{~mm}$ without saturation. The applied field of dash line and circular points in Fig. 4 is 70 Oe. The applied field was 70 Oe when the defect depth was over $6 \mathrm{~mm}$. We obtained the defect depth dependence of the light intensity without saturation. Figure 6 shows result of the comparison of defect depth measured by the dial gauge and estimated by the MO imaging. The estimated depth by the MO imaging showed good agreement with the dial gauge measurement. In this study, we obtained that the resolution of the estimated defect depth was $2.39 \mathrm{~mm}$ : the maximum error value of the results was $0.47 \mathrm{~mm}$. As a result, the defect depth can be estimated from simulated result. 


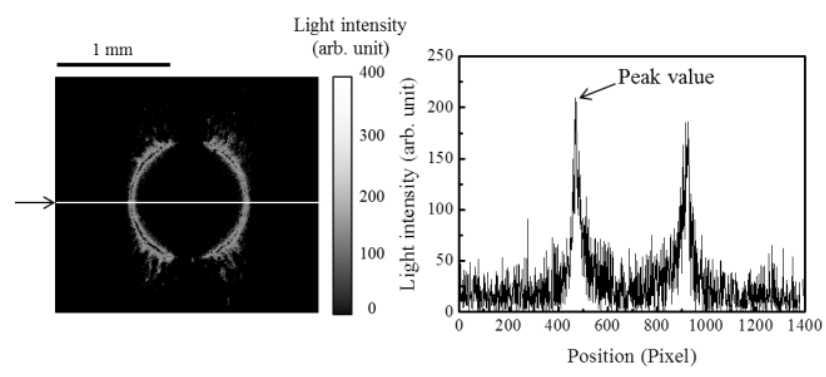

Fig. 3 Experimental results. (a) MO image of $1 \mathrm{~mm}$ depth defect. (b) Relationship between light intensity and position on a white line of (a).

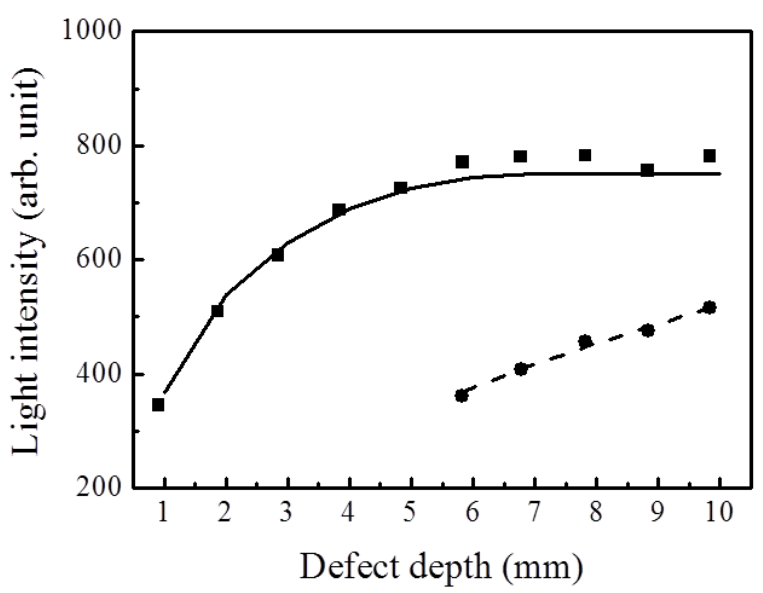

Fig. 4 The peak values of light intensity from MO image of each defect depth. The solid and dash lines show the simulation results. The solid and dash lines show when the applied field was $220 \mathrm{Oe}$ and $70 \mathrm{Oe}$, respectively. The points show the experimental results. The square and circular points show when the applied field was 220 Oe and 70 Oe, respectively.

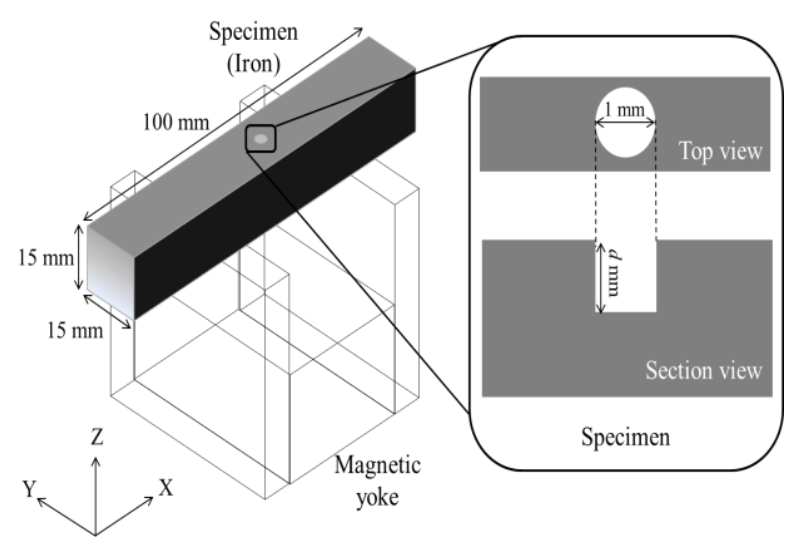

Fig. 5 A schematic of a simulation model.

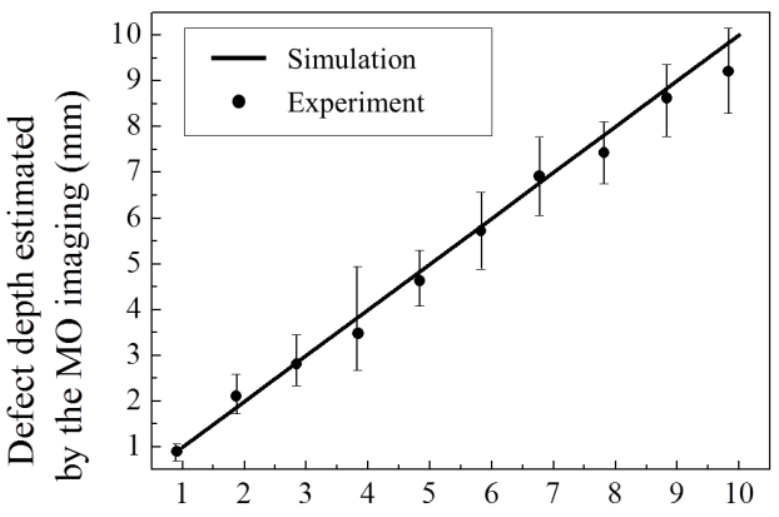

Defect depth measured by the dial gauge $(\mathrm{mm})$

Fig. 6 The comparison of defect depth measured by the dial gauge and estimated by the MO imaging.

\section{Conclusion}

We obtained the MO images of defects, which were varied from $1 \mathrm{~mm}$ to $10 \mathrm{~mm}$, by the MPC. The defect depth was estimated from light intensity peak value of the MO images. To estimate the defect depth, we calculated the defect depth dependence of the stray field. The estimated results showed good agreement with the simulated results. However, the peak value was saturated over $5 \mathrm{~mm}$ depth. Therefore, we calculated the applied field strength to estimate the defect depth without saturation. The calculation result showed that the applied field was 70 Oe when the defect depth was over $6 \mathrm{~mm}$. The estimated results showed good agreement with the dial gauge measurement. In this study, we obtained that the resolution of the estimated defect depth was $2.39 \mathrm{~mm}$ : the maximum error value of the results was $0.47 \mathrm{~mm}$.

\section{References}

1) Y. Teshima, Y. Gotoh, and N. Takahashi: JSNDI, 61, 5227 (2012).

2) P. Novotny, P. Sajdl, and P. Machac: NDT\&E International, 37, 645 (2004)

3) P. Y. Joubert, J. Pinassaud, Sensors and Actuators A, 129, 126 (2006)

4) Z. Zeng, X. Liu, Y. Deng, L. Udpa, L. Xuan, W. C. L. Shih, and G. L. Fitzpatrick: IEEE Trans. Magn., 42, 11 (2006).

5) M. Baziljevich, D. Barness, M. Sinvani, E. Perel, A. Shaulov, and Y. Yeshurun: Rev. Sci. Instrum., 83, 083707 (2012).

6) T. Ishibashi, Z. Kuang, S. Yufune, T. Kawata, M. Oda, T. Tani, Y. Iimura, and K. Sato: J. Appl. Phys., 100, 093903 (2006).

7) M. M. Tehranchi, S. M. Hamidi, H. Eftekhari, M. Karbaschi, and M. Ranjbaran: Sensors and Actuators A, 172, 365 (2011).

8) M. Inoue, R. Fujikawa, A. Baryshev, A. Khanikaev, P. B. Lim, H. Uchida, O. Aktsipetrov, A. Fedyanin, T. Murzina, and A. Granovsky: J. Phys. D: Appl. Phys., 39, R151 (2006).

9) R. Hashimoto, H. Takagi, T. Yonezawa, K. Sakaguchi, and M. Inoue: J. Appl. Phys., 115, 17A931 (2014).

10) S. Mito, H. Sakurai, H. Takagi, A. V. Baryshev, and M. Inoue: J.Appl. Phys., 111, 07A519 (2012).

Received Nov. 03, 2014; Revised Jan. 29, 2015; Accepted Mar. 16, 2015 